\title{
LOSS-RELATED VARIABLES AS RISK FACTORS FOR COMPLICATED GRIEF:
}

\section{A PERSON-CENTERED APPROACH}

Selva Ülbe*, Özge Yüksel**, Samet Baş*, Duygu Güngör*

${ }^{\star}$ Dokuz Eylul University (Turkey)

**Izmir University of Economics (Turkey)

Although the death of a loved one has traumatic effects on the course of life, many bereaved people can succeed to adapt their changing lives after the natural process of mourning. However, sometimes some bereaved people might continue to experience considerable mourning and suffer from "complicated grief" even after the long period of time[1] When the destructive impact of complicated grief on human functionality and psychological well-being is considered, it becomes crucial to figure out the underlying mechanisms explaining why some people are more prone to experience complicated grief. In the light of literature, the loss-related factors including age of the deceased, cause of death, time since loss, and subjective closeness with deceased could be risk factors for the development of complicated grief.

\section{METHOD}

\section{$\mathrm{N}=384$ \\ (Female= 293; Male $=91$}

Age $=30.40(S D=11.85)$

People with loss of a significant person

within five years.

\begin{abstract}
1.Demographic Information Form
2. Traumatic Grief Inventory-Self Report Version (TGI)[2]

3. Impact of Event Scale-Revised (IES)[3]

4. Depression, Anxiety and Stress Scale (DASS)[4]
\end{abstract}

What are the |
|

\section{What was the aim of} the study?

The main aim of the present study is to identify subgroups among the

bereaved people via person-centered approach and to compare these groups according to the lossrelated variables and psychological outcomes

\section{RESULTS}

Using 18 items of TGI, three latent classes were found according to BIC (Bayesian Information Criteria). BIC from 1-class to 9class respectively. ( $8878.5585,7335.1306$, $6986.8065,7009.2256,7048.8293,7112.9870$, $7173.7887,7245.7287,7342.5067)$

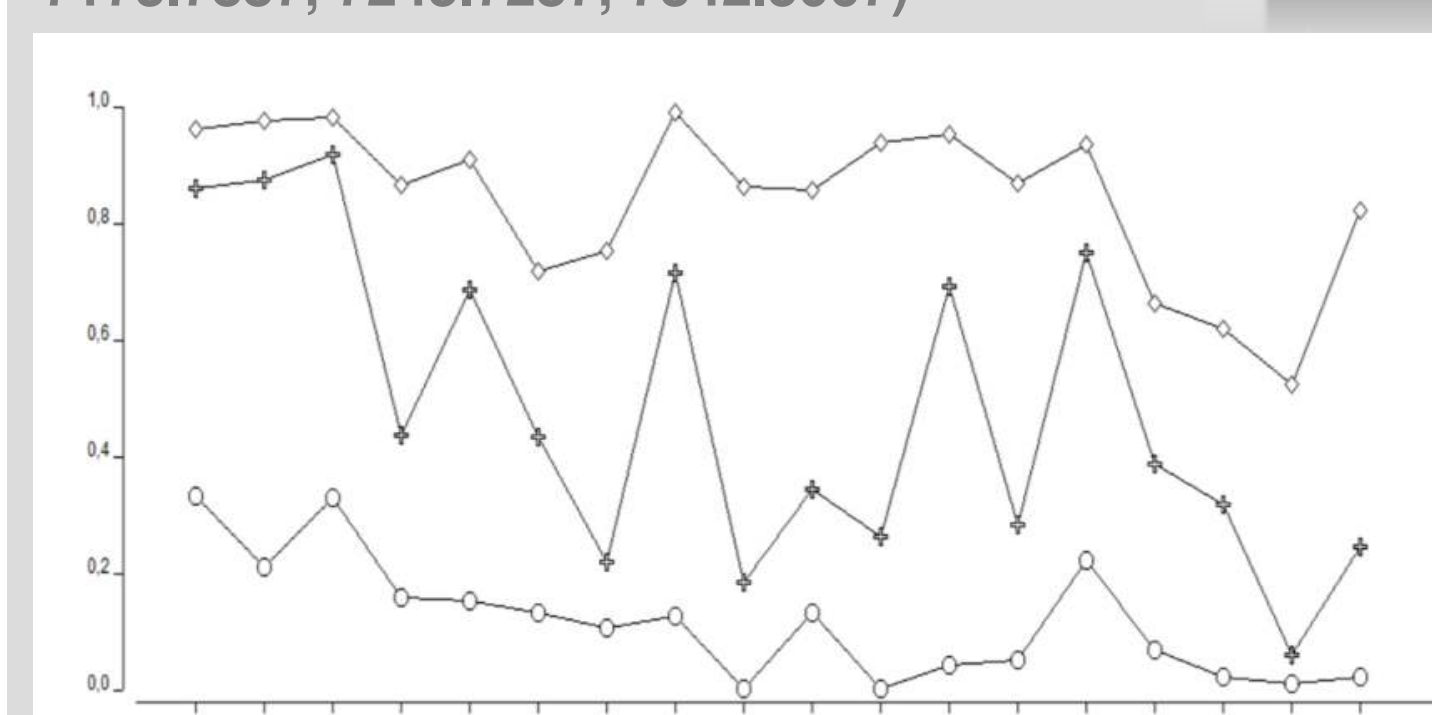

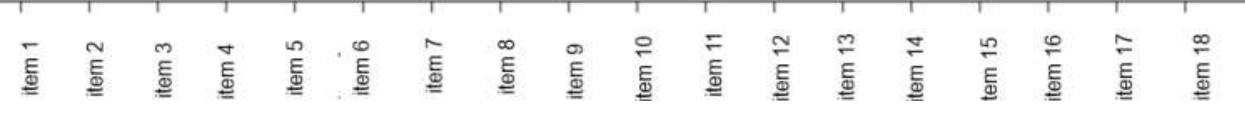

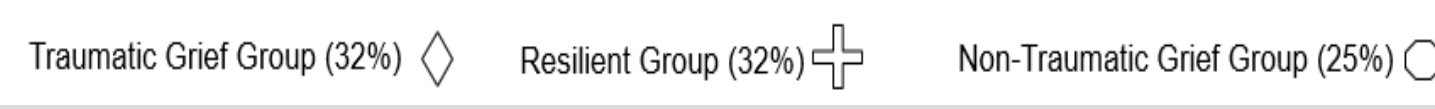
Figure 1.Estimated symptom probabilities for the three-class solution

How do the sub-groups differ from each other?

\begin{tabular}{|c|c|c|c|c|c|c|}
\hline \multirow[t]{2}{*}{ Variables } & \multicolumn{3}{|l|}{ Mean (SD) } & \multirow{2}{*}{$P$-value } & \multirow[t]{2}{*}{$F$} & \multirow{2}{*}{ Post-hoc } \\
\hline & $\begin{array}{l}R G(1) \\
(\mathrm{N}=172)\end{array}$ & $\begin{array}{l}\mathrm{TGG}(2) \\
(\mathrm{N}=120)\end{array}$ & $\begin{array}{l}\text { NTGG(3) } \\
(\mathrm{N}=92)\end{array}$ & & & \\
\hline Quality of relationship & $5.72(1.53)$ & $6.53(.99)$ & $5.24(1.63)$ & .00 & 23.16 & $3<1<2$ \\
\hline Subjective closeness & $5.73(1.51)$ & $6.56(.84)$ & $5.05(1.77)$ & .00 & 30.59 & $3<1<2$ \\
\hline Frequency of contact & $5.26(1.52)$ & $6.35(1.20)$ & $4.72(1.71)$ & .00 & 35.82 & $3<1<2$ \\
\hline Age of the deceased & $59.08(22.50)$ & $53.96(21.91)$ & $66.60(19.24)$ & .00 & 8.83 & $1=2<3$ \\
\hline Time since loss & $25.66(17.50)$ & $30.58(20.07)$ & $30.22(18.44)$ & .05 & 3.08 & $1=2=3$ \\
\hline Depresion & $13.80(5.31)$ & $18.16(5.59)$ & $10.54(3.92)$ & .00 & 59.83 & $3<1<2$ \\
\hline Anxiety & $11.87(4.49)$ & $15.98(5.39)$ & $9.84(3.87)$ & .00 & 49.72 & $3<1<2$ \\
\hline Stress & $14.59(5.05)$ & $18.15(4.99)$ & $11.71(4.34)$ & .00 & 46.62 & $3<1<2$ \\
\hline Impact of Events & $48.82(13.44)$ & $67.43(14.87)$ & $32.45(9.13)$ & .00 & 190.76 & $3<1<2$ \\
\hline Intrusion & $18.93(6.14)$ & $27.27(6.57)$ & $11.39(3.61)$ & .00 & 198.79 & $3<1<2$ \\
\hline Avoidance & $19.09(6.08)$ & $22.98(6.17)$ & $13.43(5.26)$ & .00 & 67.67 & $3<1<2$ \\
\hline Hyperarousal & $10.80(4.67)$ & 17.19(5.82) & $7.62(2.81)$ & .00 & 117.79 & $3<1<2$ \\
\hline
\end{tabular}

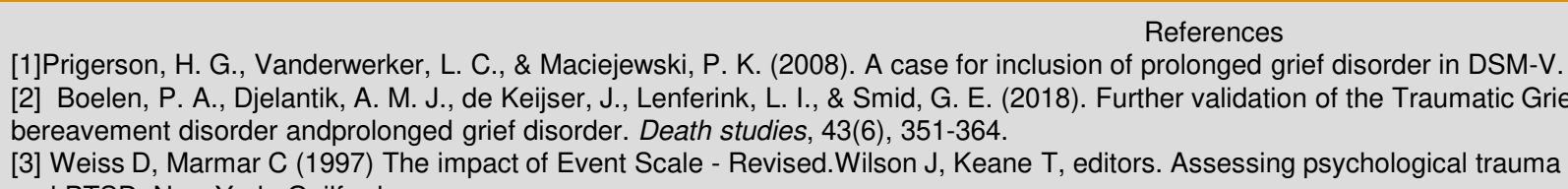

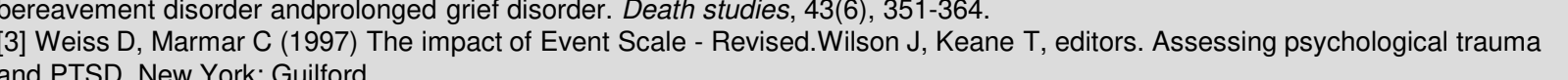

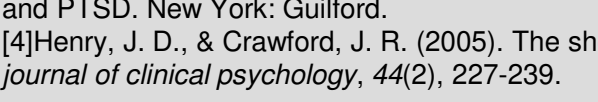

